\title{
New insights in the pathophysiology of acute myocardial infarction detectable by a contemporary troponin assay
}

\author{
Simona Ferraro a,b,*, Elia Biganzoli ${ }^{c, d}$, Giuseppe Marano ${ }^{c, d}$, Matteo Santagostino ${ }^{b}$, Patrizia Boracchi ${ }^{c, d}$, \\ Mauro Panteghini ${ }^{a}$, Angelo S. Bongo ${ }^{b}$ \\ a Laboratorio Analisi Chimico-Cliniche, A.O. 'Luigi Sacco', and Cattedra di Biochimica Clinica e Biologia Molecolare Clinica, Università degli Studi, Milano, Italy \\ b SCDO Cardiologia, Ospedale Maggiore, Novara, Italy \\ c Unit of Medical Statistics, Biometry and Bioinformatics "Giulio A. Maccacaro", Department of Clinical Sciences and Community Health, University of Milan, Italy \\ d Campus Cascina Rosa, Fondazione IRCCS Istituto Nazionale Tumori Milano, Italy
}

\section{A R T I C L E I N F O}

\section{Article history:}

Received 24 December 2012

Received in revised form 25 March 2013

Accepted 27 March 2013

Available online $\mathrm{xxxx}$

\section{Keywords:}

Myocardial pathophysiology

Analytical sensitivity

Assay

Biological interaction

Troponin

\section{A B S T R A C T}

Objectives: ST-elevation and non-ST-elevation myocardial infarction (STEMI, NSTEMI) are considered two distinct pathophysiologic entities. We evaluated cardiac troponin I (cTnI) release in STEMI and NSTEMI using a "contemporary" (CV > 10 to 20\% at the 99th percentile concentration) cTnI assay for patients undergoing early percutaneous coronary intervention $(\mathrm{PCI})$.

Design and methods: 856 patients with suspected acute coronary syndrome consecutively admitted to the Emergency Department of the Maggiore Hospital of Novara (225 STEMI and 135 NSTEMI) were selected according to: 1 ) early ( $\leq 4 \mathrm{~h}$ from admission) and successful PCI; and 2) cTnI measurements at ED presentation and within $24 \mathrm{~h}$. The influence of the MI type on cTnI concentrations at baseline and after PCI as well as the velocity of cTnI [cTnI V = absolute increase (after log conversion of cTnI measurements) / delay between the two measurements] was studied by multiple regression analysis, adjusting for patient parameters.

Results: A statistically significant interaction between MI type and time from symptoms was reported on cTnI concentrations $(\mathrm{p}<0.0001)$ : STEMI and NSTEMI differed for cTnI releases at admission and after revascularization. Higher cTnI V in STEMI was detectable in patients admitted within $6 \mathrm{~h}$ from symptoms. Baseline cTnI concentrations were lower in patients with a history of coronary artery disease (CAD) and increased with aging $(\mathrm{p}<0.0001)$. In the elderly ( $>75$ years), the cTnI V was significantly increased.

Conclusion: STEMI and NSTEMI patients have different patterns and dynamics of cTnI release influenced by the interaction with time from symptoms, by aging and history of CAD.

(c) 2013 The Canadian Society of Clinical Chemists. Published by Elsevier Inc. All rights reserved.

\section{Introduction}

Several studies currently evaluate the prognostic performances of cardiovascular biomarkers measured in the acute phase of a myocardial infarction (MI) on case series including both patients with ST-elevation myocardial infarction (STEMI) and MI with non-ST-elevation at electrocardiogram (ECG) (NSTEMI) [1,2]. However the mixing of these two populations of patients contrasts with the definition of acute MI stating STEMI and NSTEMI as two distinct pathophysiologic entities according

Abbreviations: MI, acute myocardial infarction; STEMI, ST-elevation myocardial infarction; ECG, electrocardiogram; NSTEMI, no ST-elevation at electrocardiogram myocardial infarction; cTnI, cardiac troponin I; PCI, percutaneous coronary intervention; ACS, acute coronary syndrome; ED, Emergency Department; CCU, Cardiac Care Unit; CK-MB, creatine kinase MB isoenzyme; LoD, limit of detection; CVa, analytical coefficient of variation; CAD, coronary artery disease; $\mathrm{CTnI} V$, velocity of cTnI release; CI, confidence interval.

* Corresponding author at: Laboratorio Analisi Chimico-Cliniche, Ospedale 'Luigi Sacco', Via G.B. Grassi 74, Milano, Italy. Fax: + 390250319835.

E-mail address: ferraro.simona@hsacco.it (S. Ferraro). to the presence/absence of electrocardiographic ischemic changes [3]. ECG currently represents the sole clinical tool able to early catch the main difference between pathological mechanisms underlying MI types. In fact, although advancements have been made in imaging techniques (i.e., cardiac magnetic resonance), these may still fail in the assessment of heterogeneous and small infarct size in NSTEMI, resulting below the threshold of detection [4].

Biomarkers can provide important information for both STEMI and NSTEMI pathophysiologic mechanisms. Reports have suggested that STEMI and NSTEMI have different patterns of release of inflammatory and neurohormonal markers [5,6]. Inflammatory markers show a remarkable prognostic value in NSTEMI whereas cardiac troponins (cTns) are associated with better risk prediction for STEMI [7]. This may reflect differences in the pattern of cTn release between STEMI and NSTEMI and thus in the underlying biochemical and protein expression profiles.

Current generation cTn assays [8,9] have led to an appreciation that increased cTn concentrations can be associated with myocardial necrosis independent from myocardial ischemia [10]. In particular, 
the availability of sensitive cTn detection [namely "high sensitive" assays ( $\mathrm{CV} \leq 10 \%$ at the 99th percentile) and "contemporary" assays ( $C V>10$ to $20 \%$ at the 99th percentile)] [9] could contribute more relevant information about the biochemical substrates underlying STEMI and NSTEMI. Within this context, we sought to compare the average patterns and the dynamics of CTnI release in STEMI and NSTEMI patients undergoing early percutaneous coronary intervention (PCI). The second aim was to investigate the effect of patient characteristics on cTnI release occurring within the first $24 \mathrm{~h}$ from admission in each MI type.

\section{Materials and methods}

\section{Patients}

Eight hundred and fifty-six patients with chest pain and suspected acute coronary syndrome (ACS) were consecutively admitted to the Emergency Department (ED) and then to the Cardiac Care Unit (CCU) of the Maggiore Hospital of Novara from August 2006 (when a cTnI new generation assay was introduced) to January 2009. From this case series, $360 \mathrm{MI}$ patients (225 STEMI and 135 NSTEMI) were retrospectively selected according to the following criteria:

1) PCI performed early after admission (within $4 \mathrm{~h}$ ) [11,12] according to risk stratification gauged on ECG findings and clinical evaluation reported by running guidelines [12,13]. The resting 12-lead ECG was obtained within 10 min after first medical contact upon arrival of the patient in the emergency room and immediately interpreted by a qualified physician. The finding of persistent ( $>20 \mathrm{~min}$ ) ST-elevation suggested STEMI. In the absence of ST-elevation, additional recordings were obtained in symptomatic patients. NSTEMI patients were preliminary selected according to ECG criteria and/ or creatine kinase $\mathrm{MB}(\mathrm{CK}-\mathrm{MB})$ typical increase with at least one value $\geq 99$ th percentile reference limit. High-risk NSTEMI patients undergoing early reperfusion were identified according to the following criteria: a) ST-segment depression $>0.5 \mathrm{~mm}(0.05 \mathrm{mV})$ and $<1.0 \mathrm{~mm}(0.1 \mathrm{mV})$ in two or more contiguous leads, in a clinical context of typical symptoms and coexisting cardiac disorders or previous MI; b) ST-segment depression $>1 \mathrm{~mm}(0.1 \mathrm{mV})$. Only a small percentage of cases ( $2 \%)$ were not classified accounting for inconclusive data and were excluded from the case series;

2) successful PCI according to TIMI score;

3) availability of the first cTnI measurement performed at ED presentation and of a second measurement performed at CCU after PCI within $24 \mathrm{~h}$ from admission;

4) detectable $\mathrm{cTnI}$ concentrations.

Exclusion criteria were successful thrombolysis, unsuccessful PCI (TIMI from 0 to 2 at the end of the procedure) and emergency surgery.

The time from symptoms and the time elapsed between the two cTnI measurements were reported for all patients. In particular, the time from symptom onset (namely duration of pre-hospital delay) was defined as the time interval between the onset of signs and symptoms suggestive of MI and arrival in the ED (reported in h, assuming a tolerance of $\pm 0.5 \mathrm{~h}$ ). The delay time was obtained by the cardiologist at patient's ED presentation during the clinical evaluation and reported in the medical record together with other risk factors.

Hypertension was defined as systolic tension higher than $140 \mathrm{~mm} \mathrm{Hg}$ and/or diastolic tension higher than $90 \mathrm{~mm} \mathrm{Hg}$ or use of antihypertensive drugs. Diabetes was defined as fasting venous plasma glucose concentrations $\geq 7.0 \mathrm{mmol} / \mathrm{L}$ or venous plasma glucose $2 \mathrm{~h}$ after ingestion of $75 \mathrm{~g}$ oral glucose load $\geq 11.1 \mathrm{mmol} / \mathrm{L}$. Hypercholesterolemia was defined as fasting plasma cholesterol concentration $\geq 5.0 \mathrm{mmol} / \mathrm{L}$. A positive family history for coronary artery disease (CAD) was defined as presence of a CAD in a first degree family member before the age of 55 years in males and 60 years in females. Chronic renal failure was defined according to the presence of severely reduced kidney function with an estimated glomerular filtration rate $\leq 29 \mathrm{~mL} / \mathrm{min} / 1.73 \mathrm{~m}$. Coronary and peripheral vasculopathy was defined as wall narrowing resulting in ischemia or non-inflammatory vascular lumen occlusion resulting from thromboembolic disease.

The study was approved by the Institutional Review Board of the Maggiore Hospital of Novara and informed consent was obtained from all patients. Authors have complied with the World Medical Association Declaration of Helsinki regarding ethical conduct of research involving human subjects.

\section{Sample processing and measurements}

Venous blood samples were drawn via direct venipuncture into tubes containing lithium heparin to obtain after centrifugation (5 min, $1500 \mathrm{~g}$ at $4{ }^{\circ} \mathrm{C}$ ) specimens of plasma for $\mathrm{CTnI}$ determination.

cTnI was determined by Advia Centaur TnI-Ultra ${ }^{\mathrm{TM}}$ assay [8] with an analytical range of measurement of $6-50,000 \mathrm{ng} / \mathrm{L}$, a limit of blank of $6 \mathrm{ng} / \mathrm{L}$, and a 99th percentile value (cut-off) of $40 \mathrm{ng} / \mathrm{L}$. From a 6-month retrospective evaluation of the two-level control materials supplied by the manufacturer, the intra-laboratory analytical coefficient of variation (CVa) was $15 \%$ at $80 \mathrm{ng} / \mathrm{L}$ and $5 \%$ at $9000 \mathrm{ng} / \mathrm{L}$.

\section{Statistical analysis}

The patient features were reported as dichotomous and continuous variables. Accordingly, the statistically significant differences between these parameters in STEMI and NSTEMI were assessed by F exact test and Mann-Whitney test. The results were adjusted by adopting the Bonferroni correction for multiple comparisons.

The LOESS method (locally estimated scatterplot smoothing) was used to visually assess the relationship between the two variables (i.e., cTnI concentrations and time) in this large dataset where multiple trends (i.e., thin lines from dataset points to arrows) can be hard to visualize. LOESS is the most flexible non-parametric regression technique. This smoothing function, by making minimal assumptions about the relationship among variables, can capture general patterns while reducing the noise. The result of LOESS application is the solid gray line through the moving central tendency of the relationship between cTnI measurements and time. The smoothness parameter (i.e., span) was set at 0.75: this means that each smoothed value was determined through $75 \%$ of the neighboring points [14].

Descriptive analyses were employed to define the distributions of cTnI concentrations in STEMI and NSTEMI patients. Because of the skewed distributions, cTnI concentrations followed a logarithmic transformation. The marker changes in STEMI and NSTEMI patients were estimated as velocity of cTnI release (cTnI V) as follows:

[ $\log (\mathrm{cTnI}$ concentration after PCI $)-\log (\mathrm{cTnI}$ concentration at admission $)]$ /time elapsed between the two measurements.

Multiple regression analysis was used to study the dependence from MI type of:

1) cTnI concentrations detected at baseline;

2) cTnI concentrations detected after PCI;

3) $c T n I V$.

The analysis was adjusted for patient parameters as time from symptom onset, age, gender, renal failure, and history of CAD. Age and time from symptoms were included into the models as continuous variables, accounting for possible non-linear effects by restricted cubic splines [15]. In addition, the interaction between the time from symptoms and AMI type was tested. The software used is the R-library Design by Harrell (http://CRAN.R-project.org/package=rms). 


\section{Results}

Dependence of cTnI concentrations from patient features

The baseline characteristics of the studied case series were reported as dichotomous variables in Table $1 \mathrm{~A}$. Age, cTnI concentrations at baseline and after $\mathrm{PCI}, \mathrm{CTnI}$ absolute and percentage increase, time elapsed between symptom onset to ED admission and time between the two serial detections were reported as continuous variables in Table 1B. Because of the Bonferroni correction, the difference was statistically significant at the 0.05 level if the p-value was less than $0.0036(0.05 / 14)$. Thus, STEMI and NSTEMI results differed for the proportion of patients with a previous history of CAD, age, time elapsed from symptom onset, marker concentrations after PCI, and absolute and relative increase. By comparing STEMI and NSTEMI, it is relevant that there was no evidence of a difference in baseline cTnI concentrations and in elapsed time between the two serial measurements.

Because of the importance of pre-hospital delay on MI management and patient outcomes [16,17], cTnI concentrations detected at ED admission and after PCI in each group of STEMI and NSTEMI patients were reported firstly as a function of time from symptoms (Figs. 1A, $\mathrm{B}$ ) and then as a function of time from ED arrival (time $=0$ for the first cTnI sample drawn at presentation Figs. 2A, B). In Figs. 1 and 2 solid gray lines indicated regression lines obtained by the locally weighted method LOESS [14]. According to both figures, the shape of the averaged trajectory derived from all STEMI and NSTEMI described two different saturation curves: in STEMI a faster cTnI increase than that in NSTEMI was observed.

The multivariate models investigating the effects of patient features on baseline cTnI concentrations showed a statistically significant biological interaction between MI type and time from symptoms ( $p<0.0001$, Fig. 3 ). In this context, a biological interaction is defined as the joint effect of two variables modulating cTnI release. From this result, STEMI and NSTEMI differed for the cTnI release at admission and before performing revascularization. In particular, by selecting 4 early and late time windows of clinical relevance, the ratio between mean cTnI concentrations in STEMI and NSTEMI was found to increase with the increase of the time from symptoms. Thus, mean cTnI concentrations in STEMI vs NSTEMI increased by:

1) 0.9 -fold (95\% CI: $0.5-1.5)$ at $3 \mathrm{~h}$ from symptoms to ED admission;

2) 1.4 -fold (95\% CI: $0.9-2.1)$ at $6 \mathrm{~h}$;

3) 1.8 -fold (95\% CI: $1.2-2.8)$ at $8 \mathrm{~h}$;

4) 3.3 -fold (95\% CI: $1.9-5.9)$ at $12 \mathrm{~h}$.

It was not marginal to highlight that the ratio within 5 h was nearly 1 .

In addition, cTnI baseline concentrations were influenced by the presence of a previous history of CAD. In these patients, cTnI mean concentrations were lower than those measured in patients without a previous CAD (ratio between mean concentrations of $0.45,95 \% \mathrm{CI}$ : $0.28-0.74, \mathrm{p}<0.0001)$.

A slight increase of cTnI concentrations with age increase was reported: patients 20 years older had cTnI 1.6 -fold higher $(95 \% \mathrm{CI}$ : $1.2-2.2, \mathrm{p}<0.0001)$. No significant relationships with marker concentrations were observed for gender and renal failure.

Concerning the first cTnI concentration detected after PCI within $24 \mathrm{~h}$ from admission, STEMI and NSTEMI had a different pattern of cTnI release. Also in this case the multivariate model showed a statistically significant biological interaction between MI type and time from symptoms ( $p<0.0001$, Fig. 4). According to this result, STEMI and NSTEMI had different amounts of cTnI release after revascularization and the ratio of respective CTnI mean concentrations decreased with the increase of the time from symptoms. In particular, by selecting four time windows of clinical relevance after $\mathrm{PCI}$, cTnI concentrations in STEMI vs NSTEMI increased by:

1) 14.3 -fold (95\% CI: 6.0-34.1) at $6 \mathrm{~h}$ from symptoms;

2) 10.0 -fold ( $95 \% \mathrm{CI}: 5.5-18.2)$ at $12 \mathrm{~h}$ from symptoms;

3) 5.0 -fold ( $95 \% \mathrm{CI}: 3.2-7.7)$ at $24 \mathrm{~h}$ from symptoms;

4) 2.5 -fold (95\% CI: 0.9-6.3) at $36 \mathrm{~h}$ from symptoms.

In addition, patients with a past history of CAD had cTnI mean concentrations 0.6 -fold (95\% CI: 0.4-0.9, p = 0.02) lower than patients without. No significant relationships with marker concentrations were observed for age, gender and renal failure.

Table 1

Patient characteristics reported as dichotomous (A) and continuous (B) data

\begin{tabular}{|c|c|c|c|}
\hline Variable & $\begin{array}{l}\text { STEMI } \\
(\mathrm{n}=225)\end{array}$ & $\begin{array}{l}\text { NSTEMI } \\
(\mathrm{n}=135)\end{array}$ & p-Value \\
\hline \multicolumn{4}{|l|}{$A$} \\
\hline Gender: M, n (\%) & $166(73.8 \%)$ & $87(64.4 \%)$ & NS \\
\hline Hypertension & $110(48.9 \%)$ & $80(59.3 \%)$ & NS \\
\hline Smokers & $114(50.7 \%)$ & $51(37.8 \%)$ & NS \\
\hline Diabetes & $34(15.1 \%)$ & $30(22.2 \%)$ & NS \\
\hline Vasculopathy & $18(8.0 \%)$ & $13(9.6 \%)$ & NS \\
\hline Renal failure & $9(4.0 \%)$ & $5(3.7 \%)$ & NS \\
\hline Family history of CAD & $54(24.0 \%)$ & $30(22.2 \%)$ & NS \\
\hline Hypercholesterolemia & $86(38.2 \%)$ & $50(37.0 \%)$ & NS \\
\hline Previous history of CAD & $34(15.1 \%)$ & $39(28.9 \%)$ & 0.002 \\
\hline \multicolumn{4}{|l|}{$B$} \\
\hline Age, median, 25th-75th percentiles (range) years & $64,53-74(30-98)$ & $69,60-76(39-94)$ & 0.001 \\
\hline Time from symptoms to ED admission, median (25th-75th percentiles) & $3.0(2.0,6.0)$ & $8.0(3.0,12.0)$ & $<0.0001$ \\
\hline $\begin{array}{l}\text { Time elapsed between cTnI measurement at ED admission and post-PCI cTnI measurement, median } \\
\text { (25th-75th percentiles) }\end{array}$ & $14.0(9.0,18.0)$ & $11.5(7.5,17.0)$ & NS \\
\hline cTnI concentrations at ED admission (ng/L), median (25th-75th percentiles) & $150(50,880)$ & $200(50,910)$ & NS \\
\hline cTnI concentrations after PCI (ng/L), median (25th-75th percentiles) & $47.72(14.78,128.90) \times 10^{3}$ & $6.87(1.99,20.94) \times 10^{3}$ & $<0.0001$ \\
\hline cTnI absolute increase between the two serial measurements (ng/L), median (25th-75th percentiles) & $46.32(12.00,124.20) \times 10^{3}$ & $5.16(1.13,15.13) \times 10^{3}$ & $<0.0001$ \\
\hline $\begin{array}{l}\text { \% cTnI increase between the two serial measurements (with respect to ED measurement) }{ }^{\mathrm{a}} \text {, median } \\
\text { (25th-75th percentiles) }\end{array}$ & $19,640(3281,109900)$ & $2012(242,9445)$ & $<0.0001$ \\
\hline
\end{tabular}

Legend: NS = not significant.

a Estimated as follows: absolute increase $\times 100$ / concentration at ED in each STEMI/NSTEMI patient. Report of median and 25 th-75th percentiles of the distribution of the \% increases. 


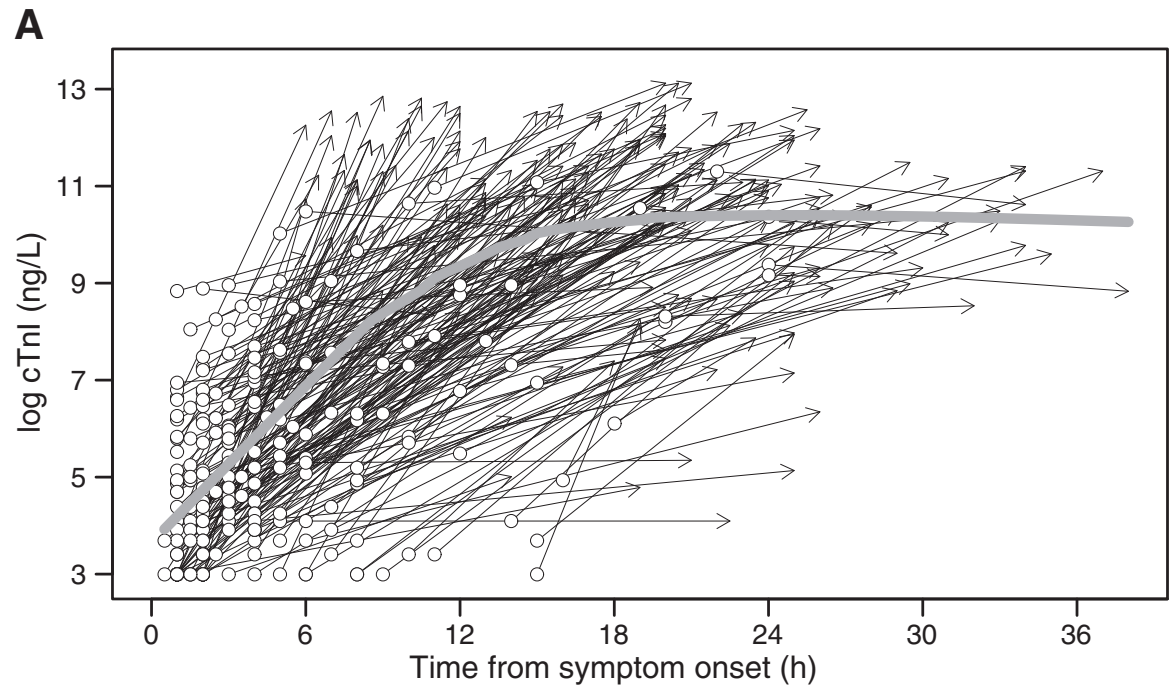

B

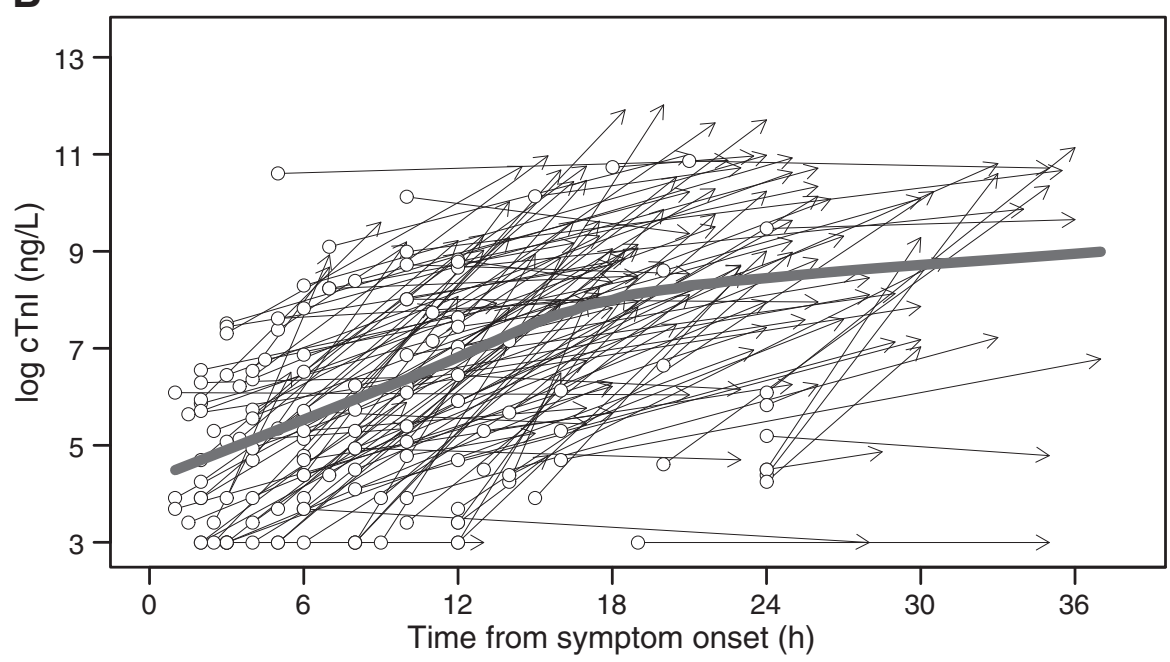

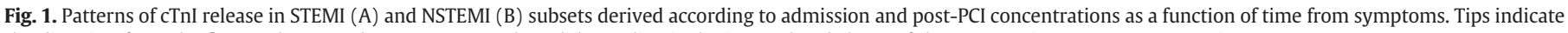
the direction from the first to the second measurement. The solid gray line is the interpolated shape of the patterns in STEMI/NSTEMI patients.

cTnI velocity

In STEMI patients, cTnI V was significantly higher $(\mathrm{p}<0.0001)$ than that of NSTEMI patients, defined as (STEMI cTnI V) - (NSTEMI cTnI V). According to the multivariable model, this difference was found to be affected by the time elapsed from symptoms (Fig. 5A), but in this case no biological interaction resulted as significant. Therefore by considering only patients admitted within $8 \mathrm{~h}$ (over this time it was not statistically significant) the difference ranged from 0.2 for those admitted early (at $3 \mathrm{~h}$ from symptoms) to 0.1 for those admitted later (at about $8 \mathrm{~h}$ ). In particular, the difference was:

1) 0.2 (95\% CI: $0.1-0.3$ ) for patients admitted at $3 \mathrm{~h}$ from symptoms to ED admission;

2) 0.1 (95\% CI: 0.1-0.2) for patients admitted at $6 \mathrm{~h}$;

3) 0.1 (95\% CI: $0.0-0.2)$ for patients admitted at $8 \mathrm{~h}$;

4) 0.0 (95\% CI: 0.0-0.1) for patients admitted at $12 \mathrm{~h}$.

In addition also the effect of age on cTnI $V$ was statistically significant ( $p=0.026$ ) with a non-linear contribution ( $p=0.01$, Fig. 5B). However, using 60 years as a threshold level for age, neither the difference between cTnI Vs at 40 and at 60 years $(0.09,95 \% \mathrm{CI}: 0.0-0.2)$ nor the difference between cTnI Vs at 75 and at 60 years $(0.04$, 95\%CI: 0.0-0.1) was statistically significant.
The difference was statistically significant only for patients older than 75 years $(>0.04)$ and tended to increase with increasing age (Fig. 5B). The effect of CAD on cTnI V was not statistically significant.

\section{Discussion}

There is a growing body of evidence that different pathophysiologic mechanisms are involved in STEMI and NSTEMI. This is reinforced by data on some cardiovascular biomarkers assuming distinct patterns of release in each MI type [5-7]. It is also noteworthy that plaque disruption, thrombosis, coronary artery occlusion and myocardial necrosis heavily differ greatly in persistence, degree and presence in STEMI and NSTEMI [18]. Although suggestive preliminary data on the different behaviors of inflammatory markers, necrosis markers and neurohormonal biomarkers are available, strong evidence about specific biochemical substrates characterizing STEMI and NSTEMI is still lacking [5-7,19].

Currently, because of the relevant improvement of analytical sensitivity, cTns are the main candidate markers to introduce new pathophysiologic insights in the heterogeneous framework of MI. Assays with higher analytical sensitivity enable the measurement of cTn concentrations that are undetectable with previous generation assays. In particular "highly sensitive" assays, meeting guideline 
A

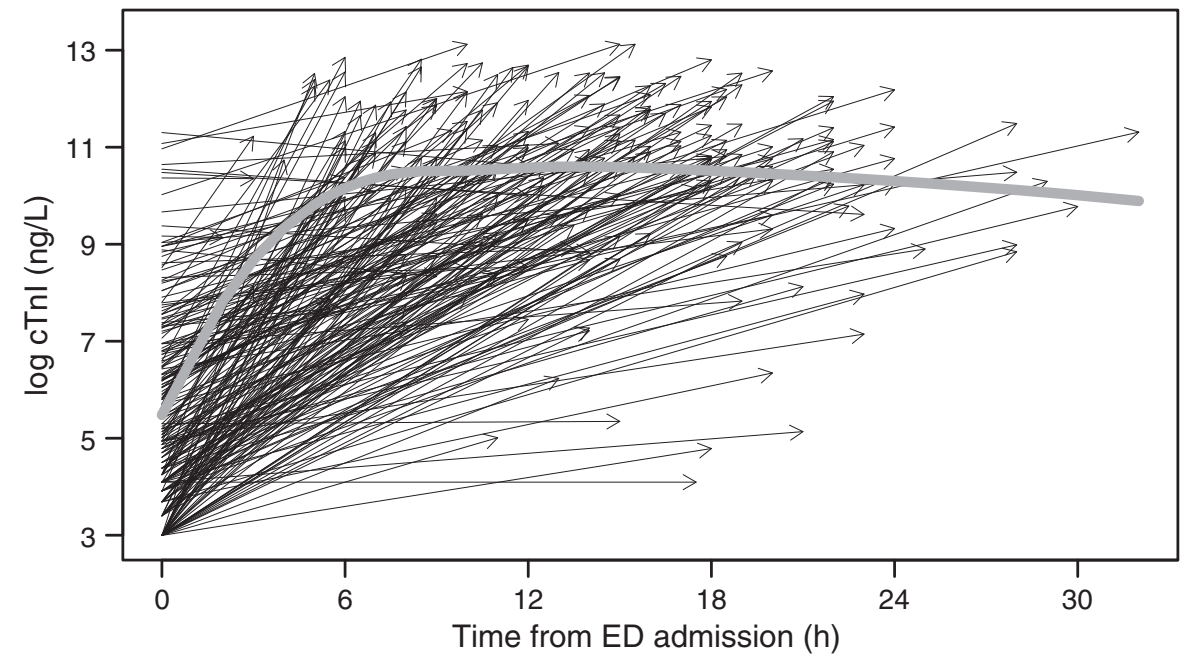

B

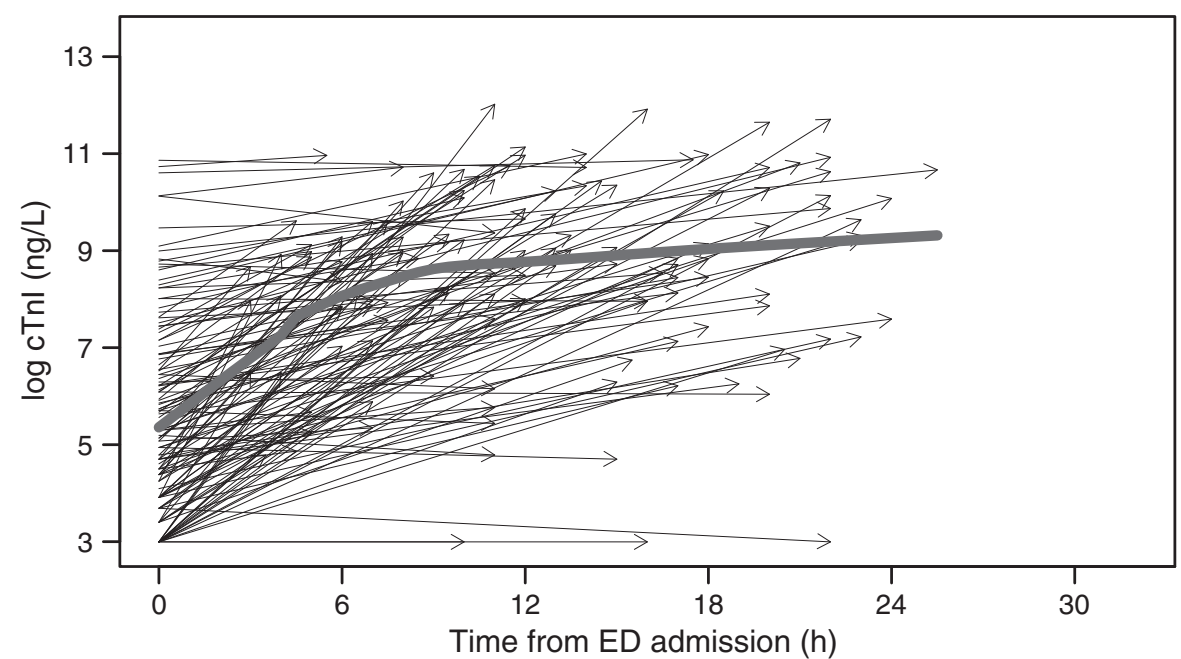

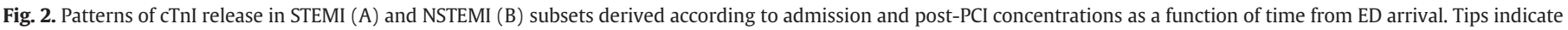
the direction from the first to the second measurement. The gray line is the interpolated shape of the patterns in STEMI/NSTEMI patients.

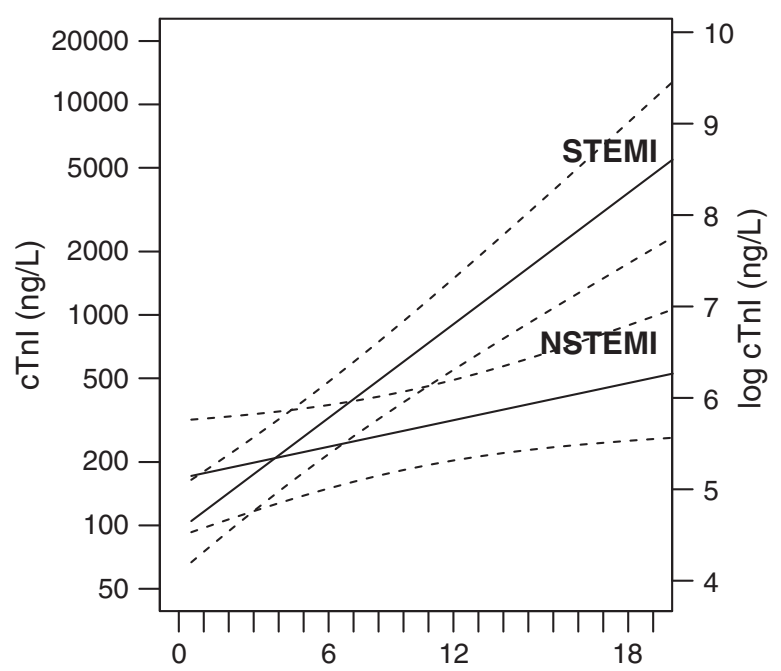

Time from symptom onset to ED admission (h)

Fig. 3. Effect of time from symptoms to ED admission on cTnI concentrations at ED admission of STEMI and NSTEMI patients estimated from the regression model. requirements (i.e., $\mathrm{CV} \leq 10 \%$ at the 99th percentile), allow the detection of $>50 \%-95 \%$ of measurable normal values below the 99th percentile threshold, whereas a lower percentage (i.e., $<50 \%$ ) may be detected by "contemporary assays" (i.e., CV $>10$ to $20 \%$ at the 99 th percentile). Anyway, contemporary assays, defined as "clinically usable", should gain the capability to measure cTn concentrations allowing the characterization of myocardial necrosis early from the onset of MI and even in the absence of overt ischemic disease [10]. In fact, the general improvement of analytical sensitivity has sparked marked debate and discussion regarding the definition of MI and even replacing the concept of traditional decision threshold with "decision marker increase" for diagnosis [20]. In the present study, we have exploited the increased sensitivity of a contemporary assay [8] to compare the patterns of cTnI release between STEMI and NSTEMI patients that have undergone early reperfusion after admission in the evolution of the acute event to catch differences in the biochemical substrates. In addition, the possible modulatory effects of patient features on cTnI releases have been investigated. The observed different shapes of $\mathrm{cTnI}$ release curves between MI types suggested a more rapid change in cTnI concentrations for STEMI with respect to NSTEMI, in addition to the well known higher increase. In particular, we have emphasized the relevance to report the effect of the 


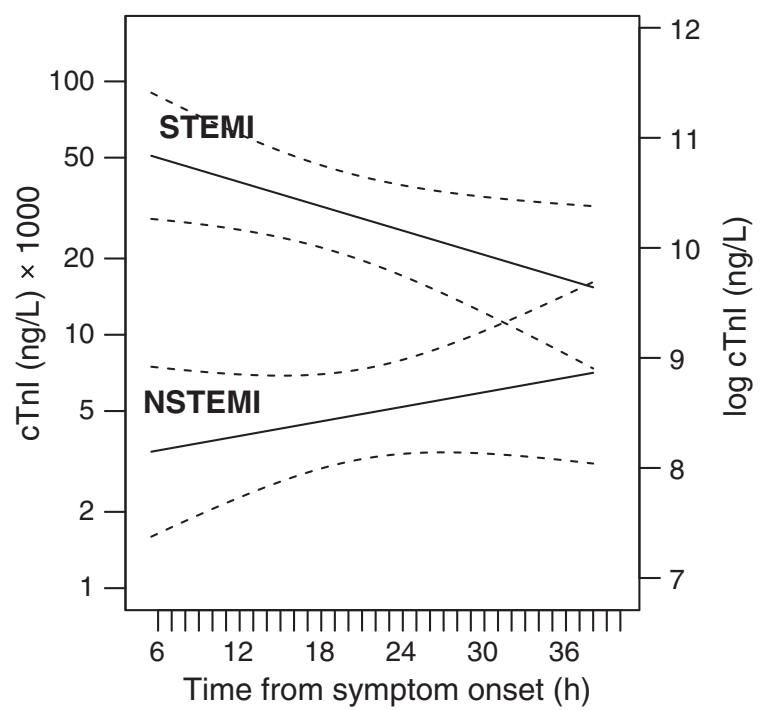

Fig. 4. Effect of time from symptoms on $\mathrm{CTnI}$ concentrations measured at $\mathrm{CCU}$ after $\mathrm{PCI}$ in STEMI and NSTEMI patients estimated from the regression model.

time from symptom onset (time from ED presentation added of pre-hospital delay) on marker release. In fact, in these patients a wide body of literature has shown that the length of pre-hospital delay strongly affects morbidity and mortality rates and it is equally associated with successful reperfusion $[16,21,22]$. In addition, international guidelines recommend that reperfusion therapy should be performed within $90 \mathrm{~min}$ after the onset of symptoms [23]. A recent systematic review has shown the reliability of reporting pre-hospital delay and the effect of patient features on it [24]. On the other hand, studies comparing yearly trends of reported pre-hospital delay have interestingly remarked that average delay times have remained essentially unchanged during the past two decades [25].

We have evidenced by showing a biological interaction that the time from symptom onset to ED admission differently modulated the pattern of marker release in STEMI and NSTEMI. For instance, cTnI concentrations at admission may appear quite similar in STEMI and NSTEMI independent of the time from symptoms. Conversely, the biological interaction between time from symptoms and MI type has revealed that cTnI concentrations on admission significantly rose in STEMI with respect to NSTEMI in those patients presenting later at ED (after about $5 \mathrm{~h}$ from acute symptoms). This should reflect a more rapid progression of necrosis in STEMI, supporting the idea that an early revascularization in these patients is mandatory to avoid the increasing loss of viable myocytes. Aside from the association of longer ischemic times with more myocardial damage and adverse clinical consequences, the effectiveness of reperfusion therapy has been shown to depend on time from symptom onset to ED presentation, with the therapy having greatest benefit for patients who present with the shortest delay [17]. Indeed, by comparing the trends of cTnI release after revascularization it is relevant to highlight a pattern of rapid increase and subsequent fast decrease that occurs only with STEMI. Conversely, the trend in NSTEMI mirrored an ongoing slow marker increase at least up to $36 \mathrm{~h}$ from chest pain. In particular, patients with early revascularization had higher cTnI concentrations at $6 \mathrm{~h}$ from symptom onset when suffering for STEMI than for NSTEMI. This difference tended to disappear with longer delays from symptom onset and thus from revascularization.

The patterns of cTnI described in STEMI patients before and after revascularization were rather expected in view of some old experimental and clinical data aiming to elucidate the relationship between the release of necrosis markers and the efficiency of reperfusion [26-29]. One study by Remppis et al. [28] on patients with STEMI (i.e., developing $\mathrm{Q}$ wave $\mathrm{MI}$ ) reported a strict relationship between successful reperfusion and highest and fast cTnT increase. In these patients, cTnT concentrations peaked at nearly $14 \mathrm{~h}$ from symptom onset and rapidly decreased, but being detectable up to the following $38 \mathrm{~h}$. Similarly, other studies described a rapid rise and decrease of CK-MB isoenzyme, myoglobin and cTn concentrations in a STEMI model despite different times to peak and rates of protein clearance from circulation. It was hypothesized that patients who underwent early and successful reperfusion with such a pattern of release had a sharp washout of necrosis markers [26-29]. In particular, a ratio between cTnT concentrations detected at $14 \mathrm{~h}$ and at $38 \mathrm{~h}$ from symptoms exceeding 1.09 appeared predictive for a successful revascularization in patients who were treated early $(<4 \mathrm{~h}$ from symptoms). In contrast, borderline cTnT ratios corresponded to either a poor or a late reperfusion or both [28].
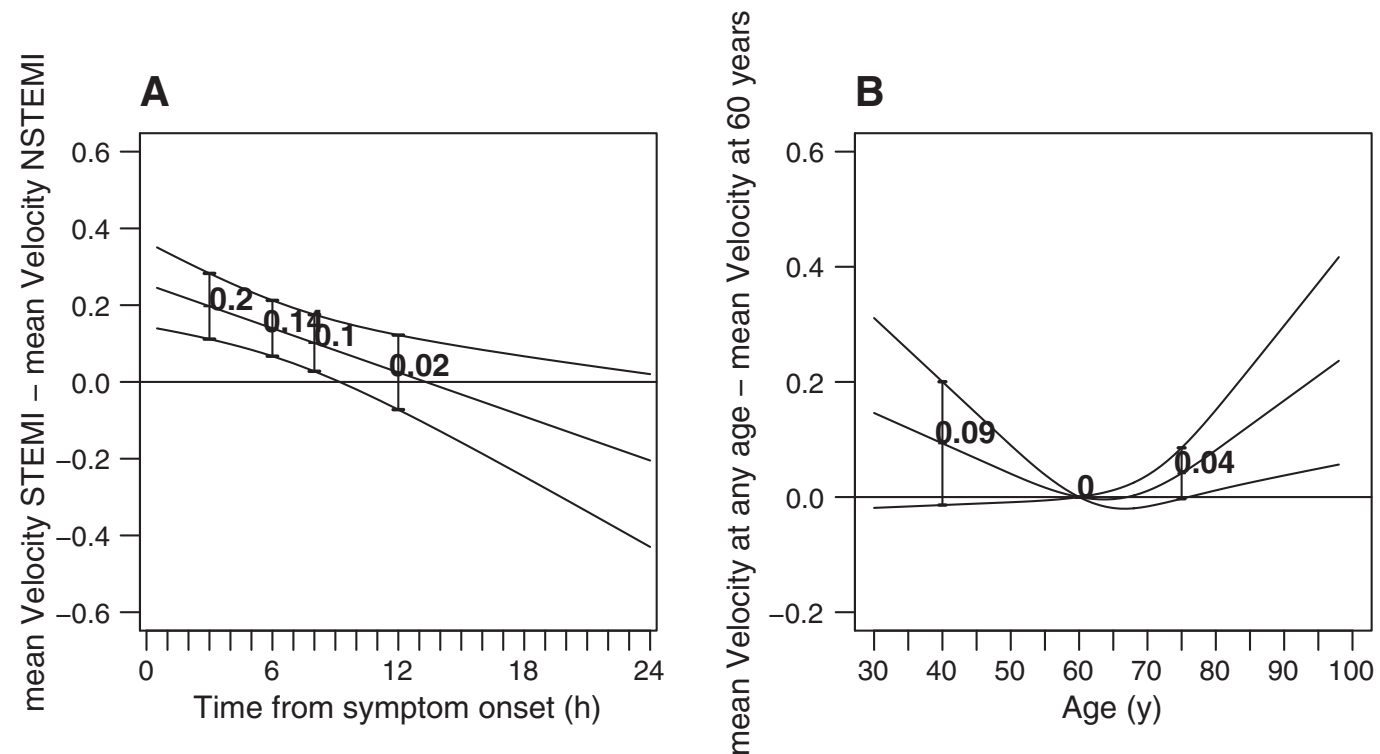

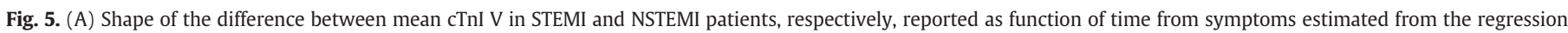
model. (B) Shape of the difference between mean cTnI V for each age with respect to 60 years as the reference value estimated from the regression model. 
The hypothesis that in NSTEMI the pattern of cTnI release after PCI may be associated with underperfused myocardium areas seemed rather questionable [27]. It is likely that the prolonged ischemia triggers tissue response that progressively reduces tissue perfusion after revascularization [30]. For NSTEMI, a PCI performed later from symptoms than in STEMI appeared to be the main cause of the slow ongoing cTnI increase that persisted for several hours. In contrast to the sharp washout of necrosis markers occurring in STEMI due to a large vessel reopening within a short time period, a different washout may characterize NSTEMI. In fact, in heterogeneous infarction the reperfusion of the microcirculation may be uneven and variable over time [30]. This is likely to influence the pattern of release of necrosis markers. In addition, we should take into account that cTnI release after PCI may be further increased by the reperfusion injury, namely irreversible damage to ischemic but viable tissue occurring with the restoration of blood flow after coronary occlusion [31]. Although it is difficult to distinguish the effect of washout and of reperfusion damage on $\mathrm{cTnI}$ release, a recent study has reported that myocardial salvage and reperfusion injury are more favorable in patients with NSTEMI than in those with STEMI only when early invasive intervention is undertaken [32]. This finding may further reinforce the hypothesis that persistent high cTnI concentrations in NSTEMI are likely due to late reperfusion with respect to the onset of chest pain.

The critical and complex influence of the time from symptoms on cTnI release in both MI types has suggested that a dynamic index such as the cTnI V might gather more consistent information on the evolution of the myocardial injury compared to a simple measure of marker increase after revascularization.

STEMI and NSTEMI patients tend to have quite similar cTnI V when admitted late. Probably cTnI concentrations measured later from symptoms when necrosis is complete or just advanced are close to the saturation point of the cTnI release curve. Thus in both MI types no tendency to a further increase seemed valuable. Under these conditions, the washout of cTn appeared to be associated with a slow release that theoretically might be influenced by the activation of tissue response initiated by prolonged ischemia for both STEMI and NSTEMI [30].

In comparing $\mathrm{CTnI}$ releases between MI types, we should take into account the fact that results cannot be generalized to overall NSTEMI patients, since we selected only those requiring urgent revascularization and classified at a higher risk.

In addition to the time from symptoms, the critical effects exerted by age and history of CAD in modulating the patterns of cTnI release may significantly contribute to marker concentrations and lead to incorrect interpretation if not taken into consideration. As expected [33,34], cTnI concentrations measured at admission for both STEMI and NSTEMI patients tended to rise with increasing age. Interestingly, the velocity of $\mathrm{cTnI}$ release significantly increased in the elderly, but only over the age of 75 years, possibly because of myocardial aging.

Patients with a history of ACS showed lower marker concentrations both at admission and after PCI than patients with a first acute event, whereas no differences in the cTnI V emerged. The condition of patients with a history of ACS could reflect the experimental model of MI developing tolerance to prolonged ischemic insult, namely ischemic preconditioning when the myocardium has been previously exposed to brief periods of ischemia and reperfusion [35]. Some experimental studies have investigated the effect of ischemic preconditioning on necrosis markers and the potential clinical interpretation [36-38]. Basically, in MI patients undergoing myocardial revascularization ischemic preconditioning was reported to reduce periprocedural cTn concentrations and to delay myocardial necrosis [36]. To date, our study is the first to suggest that in vivo ischemic preconditioning might prevent exacerbation of myocardial necrosis at the onset of a new acute event.

In conclusion, this study shows that patients with STEMI and patients with NSTEMI classified at high risk have different patterns and dynamics of cTnI release, which are further complicated by time from symptoms, aging and history of CAD. The different biochemical substrates underlying STEMI and NSTEMI should discourage the investigation of cardiovascular markers in the wide framework of MI without separating for MI type to avoid biased diagnostic/prognostic information.

\section{Conflict of interest statement}

The authors state that there are no conflicts of interest regarding the publication of this article.

Research funding: None declared.

Employment or leadership: None declared.

Honorarium: None declared.

\section{Acknowledgment}

The authors thank "Banca Popolare Di Novara" and "Fondazione Della Comunita' Novarese" for having supported the research work in cardiology.

\section{Appendix A. Supplementary data}

Supplementary data to this article can be found online at http:// dx.doi.org/10.1016/j.clinbiochem.2013.03.026.

\section{References}

[1] Kilic T, Oner G, Ural E, Yumuk Z, Sahin T, Bildirici U, et al. Comparison of the long-term prognostic value of cystatin $C$ to other indicators of renal function, markers of inflammation and systolic dysfunction among patients with acute coronary syndrome. Atherosclerosis 2009;207:552-8.

[2] Okmen E, Kasikcioglu H, Sanli A, Uyarel H, Cam N. Correlations between cardiac troponin I, cardiac troponin $\mathrm{T}$ and creatine phosphokinase $\mathrm{MB}$ elevation following successful percutaneous coronary intervention and prognostic value of each marker. J Invasive Cardiol 2005;17:63-7.

[3] Thygesen K, Alpert JS, White HD, Joint ESC/ACCF/AHA/WHF Task Force for the Redefinition of Myocardial Infarction. Universal definition of myocardial infarction. Circulation 2007;116:2634-53.

[4] Tzivoni D, Koukoui D, Guetta V, Novack L, Cowing G, CASTEMI Study Investigators. Comparison of troponin $\mathrm{T}$ to creatine kinase and to radionuclide cardiac imaging infarct size in patients with ST-elevation myocardial infarction undergoing primary angioplasty. Am J Cardiol 2008;101:753-7.

[5] Ferraro S, Lupi A, Marano G, Rossi L, Ciardi L, Vendramin C, et al. Different patterns of NT-proBNP secretion in acute coronary syndromes. Clin Chim Acta 2009;402: 176-81.

[6] Di Stefano R, Di Bello V, Barsotti MC, Grigoratos C, Armani C, Dell'Omodarme M, et al. Inflammatory markers and cardiac function in acute coronary syndrome: difference in ST-segment elevation myocardial infarction (STEMI) and in non-STEMI models. Biomed Pharmacother 2009;63:773-80.

[7] Kuch B, von Scheidt W, Kling B, Heier M, Hoermann A, Meisinger C. Differential impact of admission C-reactive protein levels on 28-day mortality risk in patients with ST-elevation versus non-ST-elevation myocardial infarction. Am J Cardiol 2008;102:1125-30.

[8] Casals G, Filella X, Bedini JL. Evaluation of a new ultrasensitive assay for cardiac troponin I. Clin Biochem 2007;40:1406-13.

[9] Apple FS. A new season for cardiac troponin assays: it's time to keep a scorecard. Clin Chem 2009;55:1303-6.

[10] Clerico A, Fortunato A, Ripoli A, Prontera C, Zucchelli GC, Emdin M. Distribution of plasma cardiac troponin I values in healthy subjects: pathophysiological considerations. Clin Chem Lab Med 2008;46:804-8.

[11] Katritsis DG, Siontis GC, Kastrati A, van't Hof AW, Neumann FJ, Siontis KC, et al. Optimal timing of coronary angiography and potential intervention in non-ST-elevation acute coronary syndromes. Eur Heart J 2011;32:32-40.

[12] Antman EM, Hand M, Armstrong PW, Bates ER, Green LA, Halasyamani LK, et al. 2007 focused update of the ACC/AHA 2004 Guidelines for the management of patients with ST-elevation myocardial infarction: a report of the American College of Cardiology/American Heart Association Task Force on Practice Guidelines. Circulation 2008;117:296-329.

[13] Anderson JL, Adams CD, Antman EM, Bridges CR, Califf RM, Casey Jr DE, et al. ACC/AHA 2007 guidelines for the management of patients with unstable angina/non ST-elevation myocardial infarction: a report of the American College of Cardiology/American Heart Association Task Force on Practice Guidelines. Circulation 2007;116:e148-304.

[14] Cleveland WS, Grosse E, Shyu WM. Local regression models. In: Chambers JM, Hastie TJ, editors. Chapter 8 of statistical models in S. Wadsworth \& Brooks/Cole; 1992.

[15] Harrell Jr FE, Lee KL, Mark DB. Multivariable prognostic models: issues in developing models, evaluating assumptions and adequacy, and measuring and reducing errors. Stat Med 1996;15:361-87. 
[16] De Luca G, Suryapranata H, Ottervanger JP, Antman EM. Time delay to treatment and mortality in primary angioplasty for acute myocardial infarction. Circulation 2004;109:1223-5.

[17] Brodie BR, Webb J, Cox DA, Qureshi M, Kalynych A, Turco M, et al. Impact of time to treatment on myocardial reperfusion and infarct size with primary percutaneous coronary intervention for acute myocardial infarction (from the EMERALD trial). Am J Cardiol 2007;99:1680-6.

[18] Parikh SV, de Lemos JA. Biomarkers in cardiovascular disease: integrating pathophysiology into clinical practice. Am J Med Sci 2006;332:186-97.

[19] Ogawa A, Seino Y, Yamashita T, Ogata K, Takano T. Difference in elevation of $\mathrm{N}$-terminal pro-BNP and conventional cardiac markers between patients with ST elevation vs non-ST elevation acute coronary syndrome. Circ J 2006;70:1372-8.

[20] Apple FS, Smith SW, Pearce LA, Murakami MM. Delta changes for optimizing clinical specificity and 60-day risk of adverse events in patients presenting with symptoms suggestive of acute coronary syndrome utilizing the ADVIA Centaur TnI-ultra assay. Clin Biochem 2012;45:711-3.

[21] Lundergan CF, Reiner JS, Ross AM. How long is too long? Association of time delay to successful reperfusion and ventricular function outcome in acute myocardial infarction. Am Heart J 2002;144:456-62.

[22] Boersma E, Maas ACP, Deckers JW, Simoons ML. Early thrombolytic treatment in acute myocardial infarction: reappraisal of the golden hour. Lancet 1996;348:771-5.

[23] ACC/AHA. Guidelines for the management of patients with ST-elevation myocardial infarction - executive summary. A report of ACC/AHA Task Force on Practice Guidelines (writing committee to revise the 1999 Guidelines for the management of patients with acute myocardial infarction). J Am Coll Cardiol 2004;44:671-719.

[24] Nguyen HL, Saczynski JS, Gore JM, Goldberg RJ. Age and sex differences in duration of prehospital delay in patients with acute myocardial infarction: a systematic review. Circ Cardiovasc Qual Outcomes 2010;3:82-92.

[25] Saczynski JS, Yarzebski J, Lessard D, Spencer FA, Gurwitz JH, Gore JM, et al. Trends in prehospital delay in patients with acute myocardial infarction (from the Worcester Heart Attack Study). Am J Cardiol 2008;102:1589-94.

[26] Ellis AK, Saran BR. Kinetics of myoglobin release and prediction of myocardial myoglobin depletion after coronary artery reperfusion. Circulation 1989;80:676-83.

[27] Christenson RH, Ohman EM, Clemmensen P, Grande P, Toffaletti J, Silverman LM, et al. Characteristics of creatine kinase-MB and MB isoforms in serum after reperfusion in acute myocardial infarction. Clin Chem 1989;35:2179-85.
[28] Remppis A, Scheffold T, Karrer O, Zehelein J, Hamm C, Grünig E, et al. Assessment of reperfusion of the infarct zone after acute myocardial infarction by serial cardiac troponin T measurements in serum. Br Heart J 1994;71:242-8.

[29] Katus HA, Diederich KW, Scheffold T, Uellner M, Schwarz F, Kübler W. Non-invasive assessment of infarct reperfusion: the predictive power of the time to peak value of myoglobin, CKMB, and CK in serum. Eur Heart J 1988;9:619-24.

[30] Cobb FR, Bache RJ, Rivas F, Greenfield Jr JC. Local effects of acute cellular injury on regional myocardial blood flow. J Clin Invest 1976;57:1359-68.

[31] Mohanlal RW, Mauve I, Zoet AC, van der Laarse A. Reperfusion induced enzyme release: washout effect or manifestation of reperfusion damage? Cardiovasc Res 1988;22:603-10.

[32] Xu J, Song YB, Hahn JY, Chang SA, Lee SC, Choe YH, et al. Comparison of magnetic resonance imaging findings in non-ST-segment elevation versus ST-segment elevation myocardial infarction patients undergoing early invasive intervention.Int Cardiovasc Imaging 2011, http://dx.doi.org/10.1007/s10554-011-9975-2 [Epub ahead of print].

[33] Venge P, Johnston N, Lagerqvist B, Wallentin L, Lindahl B. Clinical and analytical performance of the liaison cardiac troponin I assay in unstable coronary artery disease, and the impact of age on the definition of reference limits. A FRISC-II Substudy. Clin Chem 2003;49:880-6.

[34] Vasikaran SD, Bima A, Botros M, Sikaris KA. Cardiac troponin testing in the acute care setting: ordering, reporting, and high sensitivity assays-an update from the Canadian society of clinical chemists (CSCC); the case for age related acute myocardial infarction (AMI) cut-offs. Clin Biochem 2012;45:513-4.

[35] Tomai F, Crea F, Chiariello L, Gioffrè PA. Ischemic preconditioning in humans: models, mediators, and clinical relevance. Circulation 1999;100:559-63.

[36] Jenkins DP, Pugsley WB, Alkhulaifi AM, Kemp M, Hooper J, Yellon DM. Ischaemic preconditioning reduces troponin $\mathrm{T}$ release in patients undergoing coronary artery bypass surgery. Heart 1997;77:314-8.

[37] Iliodromitis EK, Kyrzopoulos S, Paraskevaidis IA, Kolocassides KG, Adamopoulos S, Karavolias $G$, et al. Increased $C$ reactive protein and cardiac enzyme levels after coronary stent implantation. Is there protection by remote ischaemic preconditioning? Heart 2006;92:1821-6.

[38] Hong DM, Jeon Y, Lee CS, Kim HJ, Lee JM, Bahk JH, et al. Effects of remote ischemic preconditioning with postconditioning in patients undergoing off-pump coronary artery bypass surgery-randomized controlled trial. Circ J 2012;76:884-90. 